\title{
Criminologie
}

\section{La régulation familiale et les comportements violents à l'adolescence : existe-t-il des différences sexuelles ?}

\section{Annie Bélanger et Nadine Lanctôt}

Volume 38, numéro 2, automne 2005

La police en pièces détachées

URI : https://id.erudit.org/iderudit/012667ar

DOI : https://doi.org/10.7202/012667ar

Aller au sommaire du numéro

\section{Éditeur(s)}

Les Presses de l'Université de Montréal

ISSN

0316-0041 (imprimé)

1492-1367 (numérique)

Découvrir la revue

Citer cet article

Bélanger, A. \& Lanctôt, N. (2005). La régulation familiale et les comportements violents à l'adolescence : existe-t-il des différences sexuelles ? Criminologie, 38(2), 173-194. https://doi.org/10.7202/012667ar
Résumé de l'article

Cet article porte sur l'influence de la famille sur la délinquance selon le sexe. Trois questions sont abordées : l'exposition aux facteurs de risque familiaux diffère-t-elle selon le sexe ?, la nature et la force d'association de ces facteurs aux comportements violents varient-elles selon le sexe ?, et quels sont les facteurs familiaux qui expliquent le mieux les comportements violents des filles et des garçons ? Un échantillon de garçons $(n=506)$ et de filles $(n=150)$ judiciarisés et un autre d'écoliers $(n=204)$ et d'écolières $(n=198)$ de Montréal sont utilisés. Les analyses révèlent que les filles judiciarisées affichent un profil familial plus problématique que celui de leurs confrères, bien qu'elles soient plus supervisées. Chez les écoliers et les écolières, le milieu familial est très semblable. Les analyses multivariées indiquent que la supervision des garçons et des filles, quel que soit l'échantillon, est une bonne protection contre les comportements violents. Par contre, les faibles liens ont plus d'importance pour la délinquance des garçons quel que soit l'échantillon, et la déviance parentale affecte un peu plus celle des filles judiciarisées. Ainsi, les mêmes concepts proximaux peuvent être utilisés pour expliquer les comportements violents des filles et des garçons, mais il faut continuer la recherche sur les concepts plus distants. Les interventions devraient porter sur l'amélioration des pratiques disciplinaires et le renforcement des liens. 


\title{
La régulation familiale et les comportements violents à l'adolescence: existe-t-il des différences sexuelles ${ }^{1}$ ?
}

\author{
Annie Bélanger \\ Candidate au doctorat \\ École de criminologie \\ Université de Montréal \\ annie.belanger.1@umontreal.ca \\ Nadine Lanctôt \\ Professeure agrégée \\ Titulaire de la Chaire de recherche du Canada sur la délinquance \\ des adolescents et des adolescentes \\ Département de psychoéducation, Faculté de l'éducation \\ Université de Sherbrooke \\ nadine.lanctot@usherbrooke.ca
}

RÉSUmÉ - Cet article porte sur l'influence de la famille sur la délinquance selon le sexe. Trois questions sont abordées: l'exposition aux facteurs de risque familiaux diffère-t-elle selon le sexe?, la nature et la force d'association de ces facteurs aux comportements violents varient-elles selon le sexe?, et quels sont les facteurs familiaux qui expliquent le mieux les comportements violents des filles et des garçons? Un échantillon de garçons $(n=506)$ et de filles $(n=150)$ judiciarisés et un autre d'écoliers $(n=204)$ et d'écolières $(n=198)$ de Montréal sont utilisés. Les analyses révèlent que les filles judiciarisées affichent un profil familial plus problématique que celui de leurs confrères, bien qu'elles soient plus supervisées. Chez les écoliers et les écolières, le milieu familial est très semblable. Les analyses multivariées indiquent que la supervision des garçons et des filles, quel que soit l'échantillon, est une bonne protection contre les comportements violents. Par contre, les faibles liens ont plus d'importance pour la délinquance des garçons quel que soit l'échantillon, et la

1. Nous tenons à remercier le Conseil de recherche en sciences humaines du Canada (CRSH) et le Fonds québécois de la recherche sur la société et la culture (FQRSC) pour leur contribution financière à ce projet de recherche. La rédaction de cet article a également été rendue possible grâce aux bourses de rédaction du Centre international de criminologie comparée (CICC) et de la fondation Berthelet Aubin. Nous remercions également M. Le Blanc pour l'accès aux données. 
déviance parentale affecte un peu plus celle des filles judiciarisées. Ainsi, les mêmes concepts proximaux peuvent être utilisés pour expliquer les comportements violents des filles et des garçons, mais il faut continuer la recherche sur les concepts plus distants. Les interventions devraient porter sur l'amélioration des pratiques disciplinaires et le renforcement des liens.

MoTS CLÉS - famille, comportements violents, différences sexuelles, jeunes judiciarisés, facteurs de risque, régressions.

ABSTRACT - This article is on the influence of family on delinquency across gender. Three questions are investigated: does the exposure to family risk factors vary as a function of gender?, do the nature and strength of association between those factors and violent behaviours vary across gender?, and which factors best explain males' and females' violent behaviours? A sample of adjudicated adolescent males $(n=506)$ and females $(n=150)$ as well as a school-based sample of males $(n=204)$ and females $(n=198)$ of Montreal were compared. Analyses show that adjudicated females, even if they are more supervised, present a more problematic familial situation as compared to their male counterparts. For the school-based sample, the familial situation is very similar. Multivariate analyses confirm that supervision of both males and females is an important protection against violent behaviours in both samples. Nevertheless, weak ties seem to have a greater importance for boys' delinquency in both sample, and parental deviance seems to be more relevant for adjudicated girls' delinquency. The same proximal concepts should be use to explain violent behaviours of males and females, but research should investigated more seriously distant concepts. The interventions should focus on improving disciplinary practices and establishing stronger family ties.

KEY WORDS - family, violent behaviours, gender gap, adjudicated youths, risk factors, regression.

Lorsque nous tentons d'expliquer la délinquance, dans les écrits, tout comme dans l'imaginaire populaire, le milieu familial est souvent pointé du doigt. Bien que la relation entre la famille et la délinquance soit étudiée depuis longtemps et dans diverses disciplines, de nouveaux questionnements ont récemment émergé. En effet, Farrington (2002) relève qu'il est probable que les facteurs familiaux associés à la délinquance aient des effets différents selon le sexe. Ce questionnement est abordé dans deux études empiriques d'envergure, pratiquement au même moment. Toutefois, ces études arrivent à des résultats divergents. Selon Farrington et Painter (2002), la délinquance des filles est plus affectée par les facteurs de risque familiaux que celle des garçons. À l'inverse, Moffitt et al. (2001) rapportent que c'est la délinquance des garçons, plus que celle des filles, qui est liée aux facteurs de risque 
familiaux. Les résultats de ces deux études remettent en question l'influence différentielle de la famille sur la délinquance selon le sexe. Ce questionnement est à l'origine de la présente étude.

\section{L'influence de la famille sur la délinquance est-elle semblable pour les garçons et pour les filles?}

Les dimensions de la famille qui seront abordées dans cette étude sont sa structure, la déviance des parents, les liens d'attachement et les pratiques disciplinaires. Plusieurs auteurs constatent l'importance d'inclure toutes ces dimensions pour bien cerner l'influence de la famille sur la délinquance (Farrington, 2002; Le Blanc, 1992a; Le Blanc et Bouthiller, 2001).

\section{La structure}

L'étude de la structure de la famille est basée sur des variables telles que le statut socioéconomique, le nombre de déménagements ou la relation de couple (uni ou séparé). Les adolescentes et les adolescents en difficulté sont reconnus pour provenir des familles affrontant plus d'obstacles (Fréchette et Le Blanc, 1987). Par exemple, les adolescents (Farrington, 1998) et les adolescentes (Rosenbaum, 1989) qui commettent des délits proviennent majoritairement de familles éclatées. Toutefois, un certain consensus règne au sujet de l'importance relativement faible de ces facteurs de risque sur la délinquance (Johnson, 1986). En fait, d'autres dimensions, particulièrement les pratiques disciplinaires et les liens familiaux, peuvent modérer leurs effets (Fréchette et Le Blanc, 1987; Johnson, 1986; Le Blanc, 1992a; Loeber et StouthamerLoeber, 1986). Peu de recherches comparent l'influence de la structure familiale sur la délinquance des garçons et des filles. Pour celles qui s'y prêtent, les résultats sont discordants. Certaines indiquent que la structure familiale est un meilleur indicateur de la délinquance des garçons que de celle des filles (Johnson et al., 1995) alors que d'autres soutiennent le contraire (Farrington et Painter, 2002). Plus encore, d'autres rapportent que l'influence de la structure familiale sur la délinquance ne varie pas selon le sexe (Johnson, 1986; Moffitt et al., 2001). Les connaissances actuelles ne permettent pas de se prononcer clairement sur l'effet différentiel de ce facteur de risque sur la délinquance selon le sexe. 


\section{La déviance des parents}

Il est reconnu que les comportements déviants des parents influencent ceux des adolescentes et des adolescents (Cusson, 1998; Farrington, 2002; Le Blanc et al., 1998b; Loeber et Stouthamer-Loeber, 1986). Les adolescentes (Farrington, 1998) et les adolescents (Rosenbaum, 1989) délinquants ont souvent des parents déviants. Par exemple, Rosenbaum (1989) rapporte des proportions trois fois plus élevées de déviance parentale chez les adolescentes ayant eu des démêlés avec la justice $(76 \%)$ que chez celles n'en ayant pas eu (24\%). La criminalité des parents est parfois même considérée comme le meilleur indicateur de la délinquance des adolescents. Ces études se sont toutefois réalisées principalement auprès d'échantillons masculins (Farrington, 2002). Quelques-unes ont comparé l'effet de la déviance parentale sur la délinquance des filles et des garçons. Selon Burton et al. (1995), avoir des membres de sa famille qui ont un dossier criminel augmente les risques de délinquance grave, mais seulement chez les garçons. Cependant, Moffitt et al. (2001), comme Farrington et Painter (2002), observent que la criminalité des parents influencent la délinquance des garçons et celle des filles de façon similaire. Bref, l'influence de la déviance parentale sur la délinquance mérite d'être mieux définie en fonction du sexe.

\section{Les liens}

Plusieurs auteurs reconnaissent l'empreinte indéniable des liens familiaux, surtout de l'attachement, pour comprendre la délinquance (Hirschi, 1969; Canter, 1982; Le Blanc, 1992a; Loeber et Stouthamer-Loeber, 1986). Certaines études suggèrent que les garçons sont plus attachés que les filles à leurs parents (Canter, 1982; Cernkovich et Giordano, 1987; Odgers et Moretti, 2002). Ce résultat concorde avec ceux qui indiquent que les conflits avec les parents seraient plus prononcés chez les filles que chez les garçons (Cernkovich et Giordano, 1987; Le Blanc et Bouthiller, 2001). En revanche, d'autres affirment qu'il n'y a pas de différence entre les sexes quant à la force de l'attachement aux parents (Anderson et al., 1999). Il est difficile d'estimer la valeur prédictive des liens familiaux sur la délinquance selon le sexe, car il existe peu de recherches comparatives. Farrington et Painter (2002) rapportent que cette influence est similaire quel que soit le sexe. Anderson et al. (1999) montrent que l'attachement aux parents est négativement et 
fortement associé à la sévérité de la délinquance des garçons lorsque l'attachement aux amis et à l'école est contrôlé. Par contre, cette association n'existe pas pour les filles. Par ailleurs, d'autres auteurs attestent que le potentiel explicatif de l'attachement aux parents est plus important pour les filles que pour les garçons, notamment dans le cas de troubles du comportement (Odgers et Moretti, 2002). Ces divergences incitent à continuer le travail empirique entamé.

\section{Les pratiques disciplinaires}

De nombreux chercheurs soutiennent que la discipline inconsistante ou coercitive et la supervision parentale relâchée sont les dimensions les plus importantes pour comprendre la délinquance (Cusson, 1990; Farrington, 2002; Le Blanc, 1992a; Le Blanc et al., 1998b; Loeber et Stouthamer-Loeber, 1986; Patterson et al., 1992). En plus de créer un stress, la discipline coercitive favorise l'acceptation des comportements violents et effrite les liens quel que soit le sexe de l'adolescent (Carter, 2003). Quant à la supervision, lorsqu'elle est relâchée, elle affaiblit les barrières morales et augmente le goût du risque des adolescents et des adolescentes (Le Blanc et al., 1998; Steffensmeier et Allan, 1996; Xiaoming et al., 2000). La majorité des études montrent que les filles sont plus surveillées que les garçons (Cernkovich et Giordano, 1987; Xiaoming et al., 2000). Cela est associé depuis longtemps aux rôles sociaux attribués aux filles (Broidy et Agnew, 1997; Heimer et De Coster, 1999; Lanctôt et Le Blanc, 2002). Le potentiel explicatif des pratiques disciplinaires est reconnu; toutefois ses conséquences sur la délinquance en fonction du sexe sont peu documentées. Farrington et Painter (2002) établissent que le manque de supervision et la discipline sévère ou irrégulière est un facteur de prédiction de délinquance plus important pour les filles que pour les garçons. Cependant, plusieurs observent l'inverse (Burton et al., 1995; Carter, 2003; Cernkovich et Giordano, 1987; Heimer et De Coster, 1999; Moffitt et al., 2001; Rankin et Wells, 1990). Ces nuances démontrent l'importance de bien distinguer l'exposition au facteur de risque de l'effet de celui-ci sur les comportements afin de bien saisir les distinctions entre les garçons et les filles. 


\section{Vers une vision intégrée des facteurs de risque familiaux}

Parmi les études recensées, la famille est rarement vue comme un ensemble où toutes ces dimensions interagissent et s'influencent mutuellement. Plusieurs études se concentrent sur quelques dimensions, souvent celles des conditions structurelles ou des pratiques disciplinaires. Il se trouve alors que peu d'études permettent d'établir si les filles et les garçons en difficulté proviennent de familles semblables. L'étude de Le Blanc et Bouthillier (2001) figure parmi les rares à avoir dressé un portrait global des familles d'une façon distincte pour les adolescents et les adolescentes en difficulté. Afin de généraliser les résultats, ils ont utilisé un échantillon de 656 adolescentes et adolescents judiciarisés au cours des années 1990 et quatre échantillons de jeunes conventionnels des années 1970, 1980 et 1990 qui totalisent 6368 cas.

Une typologie de la régulation familiale qui compte cinq profils a été générée : la famille adéquate, malhabile, déviante, punitive et conflictuelle. Pour les trois premiers types de famille, la représentativité des deux sexes s'équivaut. Cependant, les filles tendent à se retrouver un peu plus souvent que leurs confrères dans des familles punitives $(12 \%$ des filles judiciarisées contre $8 \%$ des garçons judiciarisés et $15 \%$ des écolières contre $4 \%$ des écoliers). Ce type de famille se caractérise par une supervision très relâchée, par un recours fréquent à des punitions, par la fragilité des liens familiaux et par la présence de conflits. Aussi, la famille conflictuelle caractérise davantage les écolières (18\%) que les écoliers (8\%). Cette famille se distingue par l'omniprésence des conflits, par la préséance des punitions sur la supervision et par la faiblesse des liens entretenus avec les parents. Dans l'ensemble, ces résultats suggèrent que les filles rapportent plus que les garçons une vie familiale ponctuée de conflits et de pratiques disciplinaires inconsistantes.

Une question est toutefois laissée en suspend : cette plus grande exposition des adolescentes à des familles punitives ou conflictuelles signifiet-elle que les facteurs de risque familiaux ont plus de poids pour expliquer la délinquance des adolescentes que celle des adolescents? À ce jour, très peu d'études ont vérifié l'influence différentielle des différentes composantes familiales sur la délinquance des filles et des garçons. 


\section{Problématique}

Selon les études recensées, il apparaît pertinent de s'intéresser au rôle de la famille dans la participation des filles et des garçons aux comportements délinquants. Comme le rapportaient récemment Farrington et Painter (2002), il est ardu de se prononcer sur l'existence d'un effet différent des facteurs familiaux selon le sexe, car cet effet sur la délinquance est parfois plus important pour les filles, parfois plus important pour les garçons et parfois semblable pour les deux sexes. Les résultats des diverses études sont difficilement comparables puisqu'elles se basent sur des mesures et des échantillons différents. Aussi, les différences sexuelles qui sont rapportées sont parfois statistiquement faibles et ne sont pas stables entre les différentes stratégies d'analyses (Moffitt et al., 2001). Enfin, rares sont les études qui abordent simultanément différentes composantes du milieu familial.

Les études recensées suggèrent que les résultats sont discordants quant à la structure de la famille, la déviance parentale et les liens familiaux. Du côté des pratiques disciplinaires, ce sont les filles qui sont le plus supervisées, mais ce sont les conduites des garçons qui sont les plus influencées par les pratiques disciplinaires inappropriées. Ces nuances démontrent l'importance d'étudier séparément l'exposition aux facteurs de risque et l'effet des facteurs de risque sur les comportements. De ce fait, les écrits consultés fournissent des pistes plus ou moins claires quant à l'influence que pourrait avoir la famille sur la délinquance selon le sexe. La plupart des études ne font pas la distinction entre l'exposition aux facteurs de risque familiaux et leurs effets sur la délinquance bien que Mears et al. (1998) soulignent l'importance de cette démarche. Selon eux, les filles et les garçons sont exposés différemment aux facteurs de risque de la délinquance et ils réagissent différemment à cette exposition. Il est donc judicieux de tenir compte de ces deux angles d'analyse pour comprendre les différences sexuelles. Ce questionnement est important, puisque certains auteurs croient que la délinquance des filles s'explique autrement que celle des garçons (Bertrand, 1979; Chesney-Lind et Shelden, 1998; DeKeseredy, 2000). Selon ce point de vue, la compréhension de la délinquance des filles nécessiterait la formulation de théories particulières, qui se distingueraient de celles qui ont été conçues pour les garçons. Or cette argumentation ne repose pas sur des études empiriques (Lanctôt et Le Blanc, 2002). Il importe donc de vérifier empiriquement le potentiel explicatif des concepts criminologiques relevant de la sphère familiale. 
À la lumière de ces constats, trois questions de recherches seront vérifiées. Premièrement, l'exposition aux facteurs de risque familiaux differe-t-elle selon le sexe? Deuxièmement, la nature et la force d'association des facteurs de risque familiaux aux comportements violents varient-elles selon le sexe? Finalement, quels sont les facteurs familiaux qui expliquent le mieux la délinquance des filles et celle des garçons?

L'intérêt sera particulièrement porté sur les comportements violents. Ces comportements ont été choisis plutôt que d'autres comportements délinquants, puisque les recherches indiquent une relation entre l'influence de la famille et ce type de comportements (Boidy et Agnew, 1997 ; Farrington, 1998; Farrington et Painter, 2002; Lavoie et al., 2002; Strauss, 1991 ; Xiaoming et al., 2000). Étant donné la diversité de conduites à risque et de comportements délinquants existants, cette étude s'attardera, pour des raisons de particularités, sur un seul comportement. Les mêmes hypothèses ont été vérifiées sur d'autres comportements (consommation de drogues, vols et conflits avec l'autorité) et des résultats semblables ont été obtenus ${ }^{2}$.

\section{Méthodologie}

Les échantillons

Les questions de recherche sont vérifiées auprès d'un échantillon d'adolescentes et d'adolescents «judiciarisés» et un autre d'écoliers et d'écolières. Des données ont été recueillies auprès de 506 adolescents et 150 adolescentes qui ont reçu une ordonnance de la Chambre de la jeunesse de Montréal en vertu de la Loi sur la protection de la jeunesse pour troubles de comportement sérieux ou en vertu de la Loi sur les jeunes contrevenants. L'âge moyen des adolescents et des adolescentes est respectivement de 15,7 ans et de 15,1 ans. Les ordonnances ont été reçues en 1992-1993 et elles donnaient lieu soit à un placement en centre de réadaptation, à une période de probation ou à un suivi externe par un intervenant.

Le second échantillon est composé de 402 écoliers du secondaire, dont 198 filles et 204 garçons. Une école régulière de la Commission scolaire de Montréal a été sélectionnée étant donné sa large population et sa position médiane sur une échelle représentant le désavantage

2. Ces résultats sont disponibles. S'adresser à la première auteure pour plus d'information. 
social et économique. La majorité des écoliers et des écolières $(\mathrm{n}=349$; $87 \%)$ fait partie de classes régulières. Une minorité $(\mathrm{n}=53 ; 13 \%)$ provient de classes conçues pour les élèves ayant un retard scolaire. Leur âge moyen est de 15 ans.

\section{Les mesures}

L'outil utilisé pour les deux échantillons est un questionnaire autorévélé, le MASPAQ (Le Blanc, 1992b). Il sert à recueillir des informations sur l'adaptation sociale et personnelle et sur l'ampleur et la nature des troubles de comportement et des activités délictueuses. Pour cette étude, seules les questions sur la famille et sur les comportements violents sont utilisées.

La variable dépendante est la fréquence des comportements violents. L'échelle, composée de 12 sujets ( $\mathrm{a}=0,87$ pour l'échantillon judiciarisé, 0,79 pour l'échantillon écolier), représente des comportements allant des menaces à l'agression physique. Chacun des sujets offre quatre choix de réponses allant de «jamais» à «très souvent». L'échelle varie de 4 à 48 , où 48 indique la fréquence la plus élevée. Les adolescents judiciarisés adoptent en moyenne 19,74 (écart type $=7,60$ ) de comportements violents. Les adolescentes en rapportent 17,72 (é. t. $=6,63)$. Du côté des écoliers et des écolières, les fréquences rapportées sont respectivement 14,36 (é. t. $=3,80$ ) et 13,34 (é. t. $=2,18$ ). Les adolescentes et les adolescents judiciarisés rapportent une fréquence plus élevée de comportements violents que les écoliers et les écolières. Quel que soit l'échantillon, ce sont les garçons qui révèlent la plus haute fréquence.

Suivent ensuite les variables indépendantes. La dépendance économique des parents est mesurée par le fait d'avoir déjà bénéficié ou non de l'assurance-emploi ou de l'aide sociale. La déviance des parents est représentée par une question au sujet de la fréquence de leur consommation d'alcool. Les choix de réponses vont de «jamais» à «toujours». La dimension des liens renvoie à l'échelle de l'attachement et à celle de l'investissement. La première est composée de 18 questions sur la communication, la perception des attitudes parentales et l'assimilation affective $(\mathrm{a}=0,85$ pour l'échantillon judiciarisé; 0,79 pour l'échantillon écolier). Elle varie de 10 à 40, 40 étant le plus grand attachement. L'échelle de l'investissement est composée de six sujets et fait référence au temps passé avec les parents à l'extérieur des repas $(\mathrm{a}=0,61$ pour 
l'échantillon judiciarisé; 0,69 pour l'échantillon écolier). Elle varie de 3 à 12,12 étant le plus haut niveau d'investissement.

Les pratiques disciplinaires renvoient à une échelle de supervision et une échelle de punitions. La première regroupe quatre questions, soit deux questions par parent. Elles concernent la connaissance des personnes et des lieux fréquentés par l'adolescent $(\mathrm{a}=0,85$ pour l'échantillon judiciarisé; 0,84 pour l'échantillon écolier). Ces sujets offrent quatre choix de réponses allant de «jamais» à «toujours». L'échelle varie de 2 à 8,8 étant le plus haut degré de supervision. L'échelle des punitions coercitives se compose de trois questions pour chacun des parents ( $\mathrm{a}=0,75$ pour l'échantillon judiciarisé; 0,80 pour l'échantillon écolier), concernant la remontrance, l'utilisation d'un langage grossier et le recours à la force physique. Quatre choix de réponses sont possibles, allant de «jamais» à «souvent». L'échelle varie de 3 à 12, 12 étant la position la plus problématique.

\section{La stratégie d'analyse}

L'ensemble des analyses est fait séparément pour les adolescents et pour les adolescentes. Aussi, les analyses conduites sur l'échantillon d'écoliers et d'écolières permettent d'établir le potentiel de généralisation des résultats observés auprès de l'échantillon judiciarisé.

L'exposition des adolescents et des adolescentes aux facteurs de risque familiaux est comparée à l'aide de tests de comparaison de moyennes ${ }^{3}$. Ensuite, des analyses corrélationnelles sont effectuées afin de connaître la force de la relation entre ces facteurs de risque et les comportements violents ${ }^{4}$. Des régressions incluant des effets d'interaction permettent par la suite de vérifier si les diverses variables indépendantes ont un effet différentiel sur la délinquance en fonction du sexe. Les termes d'interactions sont composés de variables standardisées afin de bien représenter le poids de chaque variable et de limiter la multicolinéarité. Finalement, des régressions linéaires mettent en évidence les facteurs de risque ayant le plus de poids dans l'explication de la violence manifestée par les adolescents et les adolescentes.

3. Sauf pour la variable dépendance économique, un test d'association sera fait.

4. Sauf pour la variable dépendance économique, un test-t sera fait. 


\section{Résultats}

L'exposition aux facteurs de risque familiaux differe-t-elle selon le sexe?

Le tableau 1 expose les résultats à l'aide de tests de moyennes. Les résultats sont présentés d'abord pour les adolescentes et les adolescents judiciarisés et ensuite pour les écoliers et les écolières.

TABLEAU 1

Différences sexuelles dans l'exposition aux facteurs de risque familiaux

\begin{tabular}{|c|c|c|c|}
\hline \multicolumn{4}{|c|}{$\begin{array}{l}\text { Échantillon judiciarisé } \\
\qquad(n=656)\end{array}$} \\
\hline \multirow[t]{2}{*}{ Variables } & \multicolumn{2}{|c|}{ Moyennes (é. t.) } & \multirow{2}{*}{$\begin{array}{l}\mathrm{X}^{2} / \\
\mathrm{T} \text { de Student }\end{array}$} \\
\hline & Garçons & Filles & \\
\hline Dépendance économique ${ }^{5}$ & $0,71(0,45)$ & $0,74(0,44)$ & 0,50 \\
\hline Déviance parentale & $1,99(0,75)$ & $2,11(0,81)$ & $-1,62$ \\
\hline Investissement & $7,75(1,65)$ & $7,61(1,82)$ & 0,89 \\
\hline Attachement & $26,80(5,57)$ & $24,63(6,67)$ & $3,58^{* * *}$ \\
\hline Supervision & $4,84(1,99)$ & $5,36(1,92)$ & $-2,85^{* *}$ \\
\hline Punitions coercitives & $4,07(1,55)$ & $5,14(2,41)$ & $-5,11 * * *$ \\
\hline \multicolumn{4}{|c|}{$\begin{array}{l}\text { Échantillon écolier } \\
\qquad(n=402)\end{array}$} \\
\hline \multirow[t]{2}{*}{ Variables } & \multicolumn{2}{|c|}{ Moyennes (é. t.) } & $X^{2} /$ \\
\hline & Garçons & Filles & \\
\hline Dépendance économique & $0,46(0,50)$ & $0,45(0,50)$ & 0,01 \\
\hline Déviance parentale & $1,78(0,70)$ & $1,62(0,61)$ & $2,30 *$ \\
\hline Investissement & $7,76(1,74)$ & $7,85(1,74)$ & $-0,53$ \\
\hline Attachement & $27,61(5,12)$ & $26,30(6,22)$ & $2,25^{*}$ \\
\hline Supervision & $4,95(1,86)$ & $5,05(1,94)$ & $-0,35$ \\
\hline Punitions coercitives & $4,38(1,64)$ & $4,64(1,87)$ & $-1,41$ \\
\hline
\end{tabular}

${ }^{*} \mathrm{p} \leq 0,05 * * \mathrm{p} \leq 0,01 \quad * * * \mathrm{p} \leq 0,001$

Chez les adolescentes et les adolescents judiciarisés, les analyses révèlent quelques différences dans l'exposition aux facteurs de risque familiaux. Bien que leurs familles vivent sensiblement le même niveau de dépen-

5. Bien qu'il n'existe pas de lien entre le sexe et la dépendance économique (voir chicarré dans le tableau 1), chez les adolescentes judiciarisées, la fréquence des comportements violents varie selon la présence ou non de la dépendance économique $(t=-2,47, p \leq 0,05)$. Chez celles qui vivent de la dépendance économique, la fréquence des comportements violents est plus élevée (moy. $=18,45$; é. t. $=7,23$ ) que chez celles qui n'en vivent pas (moy. $=15,86$; é. t. $=4,56)$. Cette distinction n'existe ni chez leurs confrères judiciarisés ni chez les écoliers ni chez les écolières. 
dance économique et de déviance parentale, les adolescentes judiciarisées rapportent un plus faible attachement familial. L'investissement familial ne se distingue pas selon le sexe. Les adolescentes rapportent aussi plus de supervision et plus de punitions coercitives que leurs confrères. En somme, les adolescentes judiciarisées affichent un profil familial plus à risques que celui des garçons, bien qu'elles soient un peu plus supervisées. Du côté des écoliers et des écolières, il existe plus de ressemblances que de différences entre les sexes. En effet, la dépendance économique, l'investissement, la supervision et les punitions coercitives sont semblables dans les familles des écoliers et des écolières. Les écoliers rapportent plus de déviance chez leurs parents que les écolières. Cependant, ils rapportent un plus fort attachement à leurs parents que les écolières. Ces différences sont toutefois faibles.

En somme, ces résultats indiquent que les adolescentes judiciarisées perçoivent plus d'incidents au sein de leur famille comparativement à leurs confrères. Cette adversité ne relève pas des difficultés financières ou des conduites déviantes des parents. Elle se manifeste plutôt par une certaine distance affective avec les parents et par l'exposition à des punitions coercitives comme des coups ou des paroles méprisantes.

La nature et la force d'association des facteurs de risque familiaux aux comportements violents varient-elles selon le sexe?

Les analyses précédentes ont indiqué que les adolescentes judiciarisées arborent un profil familial particulièrement vulnérable. Leur délinquance est-elle plus affectée par ce profil familial? Le tableau 2 représente les corrélations qui ont été générées afin de connaître la force d'association entre les facteurs de risque familiaux et la fréquence des comportements violents.

Pour l'échantillon judiciarisé, la déviance parentale et l'attachement ne sont pas associés à la fréquence des comportements violents des adolescents. Cependant, l'investissement familial est associé négativement et faiblement à la fréquence des comportements violents. Lorsque l'investissement est faible, les adolescents adoptent plus de comportements violents. La supervision parentale est négativement et modérément associée à la fréquence de leurs comportements violents. Une faible supervision est liée à une haute fréquence de ces comportements. Finalement, les punitions coercitives sont positivement et faiblement associées à la fréquence de leurs comportements violents. En d'autres termes, plus elles sont fréquentes, plus les adolescents adoptent des comportements violents. 
TABLEAU 2

Force d'association entre les facteurs de risque familiaux et les comportements violents

\begin{tabular}{lll}
\hline Variables $^{6}$ & \multicolumn{1}{c}{ Échantillon judiciarisé } \\
\hline & $\begin{array}{l}\text { Garçons } \\
(\mathrm{n}=506)\end{array}$ & $\begin{array}{l}\text { Filles } \\
(\mathrm{n}=150)\end{array}$ \\
\cline { 2 - 3 } & $\mathrm{r}$ & $\mathrm{r}$ \\
Déviance parentale & 0,06 & $0,31^{* * *}$ \\
Investissement & $-0,12^{* *}$ & $-0,21^{*}$ \\
Attachement & $-0,09$ & $-0,17^{*}$ \\
Supervision & $-0,23^{* * *}$ & $-0,29^{* * *}$ \\
Punitions coercitives & $0,13^{* *}$ & 0,1 \\
\hline Variables & \multicolumn{2}{c}{ Échantillon écolier } \\
\hline & Garçons & Filles \\
& $(\mathrm{n}=204)$ & $(\mathrm{n}=198)$ \\
\cline { 2 - 3 } & $\mathrm{r}$ & $\mathrm{r}$ \\
Déviance parentale & 0,06 & 0,01 \\
Investissement & $-0,24^{* * *}$ & $-0,03$ \\
Attachement & $-0,12$ & $-0,08$ \\
Supervision & $-0,19^{* *}$ & $-0,25^{* * *}$ \\
Punitions coercitives & 0,10 & 0,06 \\
\hline
\end{tabular}

* $\mathrm{p} \leq 0,05 \quad$ ** $\mathrm{p} \leq 0,01 \quad$ *** $\mathrm{p} \leq 0,001$

Chez les adolescentes judiciarisées, la déviance parentale est positivement et modérément liée à la fréquence de ces comportements. Plus celle-ci est élevée, plus les comportements violents sont élevés aussi. Les liens sont négativement et modérément associés à la fréquence de leurs comportements violents. Plus l'investissement et l'attachement sont faibles, plus la fréquence des comportements violents est élevée. La supervision parentale est négativement et modérément associée aux comportements violents de ces adolescentes. Ainsi, une supervision relâchée augmente les comportements violents. Finalement, les punitions coercitives ne sont pas directement associées à la fréquence de ces comportements. Ces résultats révèlent que seuls l'investissement et la supervision sont associés aux comportements violents quel que soit le sexe. Chez les adolescents, les punitions coercitives sont aussi associées à la fréquence des comportements violents. Chez leurs consœurs, s'ajoutent la déviance parentale et l'attachement.

Pour les écoliers et les écolières, il existe beaucoup moins de facteurs de risque familiaux associés aux comportements violents. En effet, la

6. Seules les variables ordinales et continues ont été utilisées. 
déviance parentale, l'attachement et les punitions ne sont pas associés à la fréquence des comportements violents des écoliers et des écolières. Cependant, l'investissement familial est négativement et modérément associé à la fréquence des comportements violents des écoliers, mais non à celle des écolières. Ainsi, lorsque les écoliers passent peu de temps avec leur famille, la fréquence de leurs comportements violents augmente. Il ressort également que la supervision est négativement et faiblement associée, avec une force similaire, à la fréquence des comportements violents des écoliers et des écolières. Lorsque la supervision est relâchée, les comportements violents sont plus fréquents, à la fois chez les écoliers et les écolières.

Indéniablement, certaines dimensions de la famille sont associées aux comportements délinquants. Ces dimensions varient-elles différemment pour les garçons et les filles? Le tableau 3 représente les termes d'interactions entre les facteurs de risque familiaux et le sexe.

TABLEAU 3

Termes d'interaction entre les facteurs de risques familiaux et le sexe

\begin{tabular}{lcc}
\hline Variables & $\begin{array}{c}\text { Échantillon judiciarisé } \\
(n=656)\end{array}$ & $\begin{array}{c}\text { Échantillon écolier } \\
(\mathrm{n}=402) \\
\beta\end{array}$ \\
\hline Dépendance économique & $-0,10^{*}$ & $-0,02$ \\
Déviance parentale & $-0,09 * *$ & 0,03 \\
Investissement & $-0,02$ & $-0,15 * *$ \\
Attachement & 0,02 & $-0,06$ \\
Supervision & 0,01 & $-0,04$ \\
Punitions coercitives & 0,04 & 0,05 \\
\hline
\end{tabular}

${ }^{*} p \leq 0,05 \quad * * p \leq 0,01 \quad * * * p \leq 0,001$

Au sein de l'échantillon judiciarisé, l'effet de la dépendance économique et la déviance parentale sur les comportements violents sont influencés par le sexe. Plus précisément, l'influence de la dépendance économique sur les comportements violents est plus accentuée pour les filles que les garçons ( $(=-0,10 ; p \leq 0,05)$. L'effet de la déviance parentale est également plus importante sur les comportements violents de ces adolescentes que sur ceux de leurs confrères $(ß=-0,09 ; p \leq 0,05)$. Cela revient à dire que les comportements violents des adolescentes judiciarisées sont plus influencés que ceux de leurs confrères par ces facteurs distants. Par contre, l'influence des facteurs proximaux comme les liens 
d'attachement et d'investissement et les pratiques disciplinaires (supervision et punitions) est semblable pour les adolescentes et les adolescents judiciarisés. Chez les écoliers et les écolières, seul l'investissement provoque un effet différent selon le sexe sur les comportements violents. Le temps passé en famille a plus d'influence sur les comportements violents des écoliers que ceux des écolières $(b=-0,15 ; p \leq 0,01)$. En d'autres termes, l'association entre l'investissement familial et les comportements violents est significativement différente entre les écoliers et les écolières.

Ces résultats indiquent que la plupart des facteurs de risque familiaux agissent similairement sur la participation des adolescents et des adolescentes à des comportements violents. Les caractéristiques des parents des adolescentes judiciarisées semblent toutefois occuper plus de poids dans l'émergence des comportements violents. Une analyse multivariée de l'ensemble des facteurs familiaux permettra de soutenir cette affirmation avec plus d'assurance.

\section{Quels sont les facteurs familiaux qui expliquent le mieux la délinquance des filles et celle des garçons?}

Le tableau 4 expose le potentiel explicatif des facteurs familiaux lorsque ceux-ci sont analysés sous une forme multivariée.

TABLEAU 4

Matrice de régressions multiples

\begin{tabular}{lllll}
\hline Variables & \multicolumn{2}{l}{ Échantillon } & judiciarisé & \multicolumn{2}{c}{ Échantillon écolier } \\
\hline & Garçons & Filles & Garçons & Filles \\
& $(n=506)$ & $(n=150)$ & $(n=204)$ & $(n=198)$ \\
& $\beta$ & $B$ & $\beta$ & $\beta$ \\
Dépendance économique & $-0,05$ & 0,13 & 0,05 & $-0,01$ \\
Déviance parentale & 0,03 & $0,20^{*}$ & $-0,04$ & 0,15 \\
Investissement & $-0,10^{*}$ & $-0,07$ & $-0,24^{*}$ & 0,02 \\
Attachement & 0,09 & 0,10 & 0,02 & 0,07 \\
Supervision & $-0,22^{* * *}$ & $-0,24^{*}$ & $-0,20^{*}$ & $-0,36^{* * *}$ \\
Punitions coercitives & 0,09 & 0,05 & 0,13 & 0,11 \\
$R^{2}$ & $7,5 \%$ & $15,4 \%$ & $12,5 \%$ & $10,1 \%$ \\
F & $6,12^{* * *}$ & $3,90^{* * *}$ & $2,87 *$ & $2,68^{*}$ \\
\hline
\end{tabular}

$* p \leq 0,05 \quad * * p \leq 0,01 \quad * * * p \leq 0,001$ 
Pour les garçons judiciarisés, les facteurs de risque familiaux analysés expliquent 7,5\% de la variance des comportements violents ( $\mathrm{F}=6,12$; $p \leq 0,001)$. L'investissement familial ( $(=-0,10 ; p \leq 0,05)$ et la supervision $(B=-0,22 ; p \leq 0,001)$ sont significatifs. Le premier est de faible force et le second est plutôt modéré. Ces résultats suggèrent que les adolescents judiciarisés qui passent peu de temps avec leurs parents et qui sont peu supervisés ont une fréquence plus élevée de comportements violents. Chez leurs consœurs judiciarisées, les facteurs expliquent $15,4 \%$ de la variance des comportements violents ( $F=3,90$; $\mathrm{p} \leq 0,001)$. Dans ce cas-ci, la déviance des parents $(\Omega=0,20 ; \mathrm{p} \leq 0,05)$ et la supervision $(\Omega=-0,24 ; \mathrm{p} \leq 0,05)$ justifient modérément la fréquence des comportements violents. Lorsque les adolescentes judiciarisées vivent avec des parents qui consomment régulièrement de l'alcool et qui ne sont pas en mesure de fournir une supervision appropriée, la fréquence de leurs comportements violents est élevée.

Du côté des écoliers, il apparaît que ces facteurs expliquent 12,5\% de la variance de leurs comportements violents $(\mathrm{F}=2,87 ; \mathrm{p} \leq 0,05)$. L'investissement $(~(B=-0,24 ; p \leq 0,05)$ et la supervision ( $(=-0,20$; $\mathrm{p} \leq 0,05)$ ont des relations de force modérée avec les comportements violents. Cela signifie que les écoliers qui sont peu investis par leur famille et qui font l'objet d'une supervision relâchée commettent plus de comportements violents. Chez les adolescentes, les facteurs de risque familiaux à l'étude expliquent $10,1 \%$ de la variance de leurs comportements violents $(\mathrm{F}=2,68 ; \mathrm{p} \leq 0,05)$. Parmi ces facteurs, seule la supervision prédit modérément la fréquence de leurs comportements violents $(\Omega=-0,36 ; p \leq 0,001)$. Les écolières peu supervisées par leurs parents affichent une fréquence de comportements violents élevée.

Somme toute, ces facteurs familiaux expliquent une faible proportion des comportements violents. Par ailleurs, il ressort que la supervision représente une dimension importante pour prédire les comportements violents quel que soit le sexe. Chez les garçons, qu'ils soient écoliers ou judiciarisés, l'investissement familial apparaît également important pour expliquer les comportements violents. Chez les adolescentes judiciarisées, des facteurs plus distants, comme la déviance des parents, agissent sur ces comportements. 


\section{DISCUSSION}

Tentons maintenant de répondre aux trois questions à l'origine de notre recherche. Premièrement, l'exposition aux facteurs de risque familiaux differe-t-elle selon le sexe? Deuxièmement, la nature et la force d'association des facteurs de risque familiaux aux comportements violents varientelles selon le sexe? Troisièmement, quels sont les facteurs familiaux qui expliquent le mieux la délinquance des filles et celle des garçons?

Cette étude constitue un complément psychosocial intéressant à la recherche sur la délinquance juvénile et à celle sur les interventions cliniques. En effet, le questionnement à l'origine de cette recherche, soit les résultats divergents entre l'étude de Farrington et Painter (2002) et celle de Moffitt et al. (2001), amène à se questionner sur l'effet différentiel des facteurs familiaux sur la délinquance selon le sexe.

Pour la première question, les résultats indiquent que le milieu familial des garçons et des filles, quel que soit l'échantillon, se ressemble plus qu'il ne differe. Toutefois, et comparativement aux adolescents judiciarisés, les adolescentes judiciarisées sont l'objet de plus de punitions coercitives et elles ont des liens familiaux plus ténus. Ces résultats concordent avec les études qui soutiennent que les filles délinquantes sont confrontées à des difficultés sur le plan relationnel et qu'elles vivent souvent dans des milieux où la violence sévit (Belknap et Holsinger, 1998; Broidy et Agnew, 1997; Cernkovich et Giordano, 1987; Lanctôt, 1999). Cependant, contrairement à d'autres études (Lavoie et al., 2002; Moffitt et al., 2001; Strauss, 1991), celle-ci indique que les filles reçoivent plus de punitions coercitives que les garçons. Les adolescentes judiciarisées démontrent donc un profil familial plus désavantagé que celui de leurs confrères. Il faut ensuite se questionner sur l'effet de ces ressemblances et de ces distinctions sur les comportements violents.

Pour les deuxième et troisième questions de recherche, les analyses de corrélation révèlent que la supervision constitue un facteur fortement associé à la violence, autant pour les filles que pour les garçons, et ce, quel que soit l'échantillon. Les analyses de régression ont aussi confirmé ce pouvoir d'inhibition de la supervision parentale sur les comportements délinquants. Plusieurs études avaient obtenu ce résultat spécifiquement pour les garçons (Cusson, 1990; Farrington, 2002; Le Blanc et al., 1998b; Loeber et Stouthamer-Loeber, 1986). Notre étude indique que ces résultats s'appliquent tout aussi bien aux filles. De la même façon, les analyses d'interaction, tout comme les régressions, 
montrent que les dimensions les plus proximales ont le même impact sur les comportements violents, quel que soit le sexe et l'échantillon. Ainsi, l'attachement et les pratiques disciplinaires n'ont pas d'effet différent selon le sexe sur les comportements violents.

Toutefois, des facteurs qui relèvent directement des caractéristiques des parents, dont leur dépendance économique et leur déviance, sont particulièrement associés aux comportements violents des filles judiciarisées. Ces associations ne s'observent pas pour les garçons, qu'ils soient judiciarisés ou écoliers. Les termes d'interaction confirment ces résultats. Le potentiel explicatif de la déviance parentale semble notable pour les adolescentes judiciarisées, puisque ce facteur de risque ressort aussi de l'analyse multivariée. Ce dernier résultat est contraire à ceux qui avaient été recensés à ce sujet (Burton et al., 1995; Moffitt et al., 2001; Farrington et Painter, 2002). C'est donc dire que des facteurs plus distants indiquent un potentiel explicatif supérieur pour les filles que pour les garçons. À l'inverse, l'investissement avec la famille occupe un poids explicatif plus important pour les garçons que pour les filles.

Ces résultats suggèrent que seuls les facteurs distants (ceux ayant une influence indirecte) régularisent plus fortement les conduites violentes des filles, comparées à celles des garçons. Dans la présente étude, la presque totalité des facteurs les plus proximaux expliquent aussi bien la violence des filles que celle des garçons. Par le fait même, les théories criminologiques classiques sont utiles et pertinentes à la compréhension de la violence des adolescentes. Cela rappelle l'importance d'appliquer sensiblement les mêmes théories aux garçons et aux filles et d'éviter la dichotomie dans les explications. Comme Lanctôt et Le Blanc (2002) l'ont proposé, il est préférable d'opter pour un ajustement des théories existantes pour mieux représenter la délinquance des filles plutôt que de polariser les explications selon les sexes.

Comme le suggèrent nos résultats, l'ajustement des théories existantes devrait porter sur des facteurs plus distants afin de bien saisir la réalité des filles et des garçons. En effet, la violence des adolescentes judiciarisées est directement associée aux caractéristiques de leurs parents (leur faible statut socioéconomique et leur consommation d'alcool), alors que ce lien n'a pas été observé chez les garçons. Ces caractéristiques parentales s'apparentent à ce que Broidy et Agnew (1997) associent aux conditions adverses. Par conséquent, il s'avère pertinent de porter attention à ces facteurs dans les explications de la délinquance. Les théories de l'adversité semblent une voie à emprunter et à utiliser pour 
mieux saisir les distinctions entre les comportements délinquants des filles et des garçons, notamment chez les adolescentes et les adolescents judiciarisés.

Il demeure que certaines limites peuvent atténuer ces résultats. En effet, considérant les limites liées à la mesure de nos concepts plus distants, il est difficile d'affirmer avec certitude que des facteurs familiaux distants sont davantage associés à la violence des filles qu'à celle des garçons. Notamment, la déviance parentale, mesurée par une seule question, parvient difficilement à capter la nature et l'ampleur des conduites et des attitudes déviantes des parents. De plus, les analyses gagneraient à inclure d'autres facteurs comme la victimisation sexuelle, puisque les écrits soutiennent son incidence sur la délinquance, surtout chez les filles (voir entre autres : Belknap et Holsinger, 1998; Broidy et Agnew, 1997; Chesney-Lind et Shelden, 1998). Force est d'admettre que ce facteur viendrait également altérer l'établissement des autres dimensions familiales comme les liens et les pratiques disciplinaires. Il faut également reconnaître que l'échantillon provenant des écoles avait une portée statistique plus limitée que celle des adolescentes et des adolescents judiciarisés, ceci parce que les indicateurs liés à la structure de la famille variaient peu entre les répondants. Cette plus grande homogénéité rendait difficile l'analyse des différences individuelles. Aussi, il est important de rappeler que les analyses ne sont effectuées qu'à un seul temps de mesure. Il n'est donc pas possible d'établir un ordre causal. Pour comprendre plus en détail l'impact différentiel des facteurs de risque familiaux selon le sexe, il faudrait recourir à un modèle longitudinal complet.

Ces limites mises à part, cette étude fournit des points de repère intéressants pour orienter la prévention et l'intervention. Il ressort qu'il faut d'abord cibler la supervision, puisque son influence sur les comportements violents s'est démarquée, quels que soient le sexe ou l'échantillon. Il faut également favoriser l'amélioration des interventions qui visent les caractéristiques parentales à problèmes, comme la consommation abusive d'alcool de la part des parents, et de celles qui favorisent l'établissement de forts liens. C'est donc à dire qu'il faut inclure les parents dans les programmes de réadaptation. Il apparaît plus judicieux de tenir compte de divers acteurs et de ne pas orienter l'intervention seulement sur le jeune ou les parents (Normand et al., 2000). Les parents doivent être en mesure de projeter un modèle positif et d'établir une bonne relation avec leurs enfants. Cela importe fortement chez les 
adolescentes judiciarisées pour qui l'aspect relationnel est lacunaire. Il faut aussi favoriser le recours à des punitions efficaces, à une bonne supervision et au perfectionnement de la capacité de résolution de problèmes (Cusson, 1998; Patterson et al., 1992; Reese et al., 2000).

Citons, à titre d'exemple, le programme Aggression Replacement Training (ART), appliqué dans les centres jeunesse Batshaw, qui privilégie l'intervention auprès des parents (Lanctôt et Lamoureux, 2004), des jeux de rôles, de rétroactions et de devoirs. La famille et l'adolescent travaillent ensemble afin de trouver des solutions à diverses situations qui créent des tensions (heures de rentrées, disputes, etc.). Ces activités visent donc à réduire les facteurs de stress et à affermir les liens, tout en améliorant les pratiques disciplinaires. Sachant que les parents des adolescentes judiciarisées consomment beaucoup d'alcool et recourent à des punitions coercitives, ces activités pourraient leur être utiles. Puisqu'il est clair qu'une seule et même intervention ne peut convenir à tous les adolescents (Le Blanc et al., 1998a), il serait judicieux d'approfondir nos connaissances sur les influences différentielles de la famille selon le sexe.

\section{Références}

Anderson, B. J., Holmes, M. D. \& Ostresh, E. (1999). Male and female delinquents' attachments on severity of self-reported delinquency. Criminal Justice and Behavior, 26 (4), 435-452.

Belknap, J. \& Holsinger, K. (1998). An overview of delinquent girls: How theory and practice have failed and the need for innovative changes. In R.T. Zaplin (ed.), Female offenders, critical perspectives and effective interventions (3164). Maryland: Aspen Publishers Inc.

Broidy, L. \& Agnew, R. (1997). Gender and crime: A general strain theory perspective. Journal of Research in Crime and Delinquency, 34 (3), 275-305.

Burton, V. S., Evans, T. D., Kethineni, S. R., Cullen, F. T., Dunaway, R. G., \& Payne, G. L. (1995). The impact of parental controls on delinquency. Journal of Criminal Justice, 23 (2), 111-126.

Canter, R. J. (1982). Family correlates of male and female delinquency. Criminology, 20 (2), 149-167.

Carter, H. (2003). Family strain, gender, and delinquency. Sociological Perspectives, 46 (1), 107-135.

Cernkovich, S. A. \& Giordano, P. C. (1987). Family relationships and delinquency. Criminology, 25, 285-321.

Chesney-Lind, M. \& Shelden, R. G. (1998). Girls, delinquency, and juvenile justice ( $2^{\text {nd }}$ edition). Belmont, California : West / Wadsworth. 
Cloutier, R. (1996). Psychologie de l'adolescence. Montréal: Gaëtan Morin Éditeur.

Cusson, M. (1990). Croissance et décroissance du crime. Paris : Presses universitaires de France.

Cusson, M. (1998). Criminologie actuelle. Paris: Presses universitaires de France.

DeKeseredy, W. S. (2000). Women, crime, and the criminal justice system. Cincinnati: Anderson.

Farrington, D. P. (1998). Predictors, causes, and correlates of male youth violence. In M. Tonry \& M. H. Moore (ed.), Youth Violence (421-475). Chicago, IL: University of Chicago Press.

Farrington, D. P. (2002). Families and crime. In J. Q. Wilson \& J. Petersilia (ed.), Crime: Public policies for crime control, $2^{\text {nd }}$ edition (129-148). Oakland, California: Institute for Contemporary Studies Press.

Farrington, D. P. \& Painter, K. A. (2002). Gender differences in offending: Implications for risk-focussed prevention. London: Rapport du Home Office Research Study.

Fréchette, M. \& Le Blanc, M. (1987). Délinquances et délinquants. Boucherville: Gaëtan Morin Éditeur.

Heimer, K. \& De Coster, S. (1999). The gendering of violent delinquency. Criminology, 37 (2), 277-317.

Hirschi, T. (1969). Causes of delinquency. Berkeley: University of California Press.

Johnson, R. E. (1986). Family structure and delinquency: General patterns and gender differences. Criminology, 24 (1), 65-83.

Johnson, R. A., Su, S. S., Gerstein, D. R., Shin, H. C. \& Hoffman, J. P. (1995). Parental influences on deviant behaviour in early adolescence: A logistic response analysis of age and gender differentiated effects. Journal of Quantitative Criminology, 11 (2), 167-193.

Lanctôt, N. (1999). Les perspectives théoriques sur la marginalité des adolescentes: vers une intégration des connaissances. Revue internationale de criminologie et de police technique et scientifique, 1, 31-54.

Lanctôt, N. \& Lamoureux, A. (2004). Aggression Replacement Training: The description of the implementation process at Batshaw Youth and Family Centre. Montréal: Rapport déposé pour les centres jeunesses Batshaw.

Lanctôt, N. \& Le Blanc, M. (2002). Explaining deviance by adolescent females. Crime and Justice: A review of Research, 29, 113-202.

Lavoie, F., Hébert, M., Tremblay, R., Vitaro, F., Vézina, L., \& McDuff, P. (2002). History of family dysfunction and perpetration of dating violence by adolescent boys: A longitudinal study. Journal of Adolescent Health, 30, 375-383.

Le Blanc, M. (1992a). Family dynamics, adolescent delinquency and adult criminality. Psychiatry, 55, 336-353.

Le Blanc, M. (1992b). MASPAQ, Manuel sur les mesures de l'adaptation sociale et personnelle pour les adolescents québécois. Montréal: Groupe de recherche sur l'inadaptation psychosociale à l'enfance, Université de Montréal.

Le Blanc, M. \& Bouthiller, C. (2001). Formes de la régulation familiale et conduites déviantes chez les adolescents. Revue de psychoéducation et d'orientation, 30 (2), 329-348. 
Le Blanc, M., Dionne, J., Proulx, J., Grégoire, J. \& Trudeau-Le Blanc, T. (1998a). Intervenir autrement. Un modèle différentiel pour les adolescents en difficulté. Montréal: Les Presses de l'Université de Montréal.

Le Blanc, M., McDuff, P. \& Kaspy, N. (1998b). Family and early adolescent delinquency: A comprehensive sequential family control model. Early Child Development and Care, 142, 63-91.

Loeber, R. \& Stouthamer-Loeber, M. (1986). Family factors as correlates and predictors of juvenile conduct problems and delinquency. Crime and Justice: An Annual Review of Research, 7, 29-150.

Mears, D. P., Ploeger, M. \& Warr, M. (1998). Explaining the gender gap in delinquency: Peer influence and moral evaluations of the behaviour. Journal of Research in Crime and Delinquency, 35 (3), 251-266.

Moffitt, T., Caspi, A., Rutter, M. \& Silva, P. A. (2001). Sex differences in antisocial behaviour. Cambridge, UK: Cambridge University Press.

Normand, C. L., Vitaro, F. \& Charlebois, P. (2000). Comment améliorer la participation et réduire l'attrition des participants aux programmes de prévention? In F. Vitaro \& C. Gagnon (ed.), Prévention des problèmes d'adaptation chez les enfants et les adolescents, Tome I (101-140). Montréal: Presses de l'Université du Québec.

Odgers, C. L. \& Moretti, M. M. (2002). Aggressive and antisocial girls: Research update and challenges. International Journal of Forensic Mental Health, 1 (2), 103-119.

Patterson, G. R., Reid, J. B. \& Dishion, T. J. (1992). Antisocial boys. Eugene OR: Castalia.

Rankin, J. H. \& Wells, L. E. (1990). The effect of parental attachments and direct controls on delinquency. Journal of Research in Crime and Delinquency, 27 (2), 140-165.

Reese, L. E., Vera, M. E., Simon, T. R. \& Ikeda, R. M. (2000). The role of families and care givers as risk and protective factors in preventing youth violence. Clinical Child and Family Psychology Review, 3 (1), 61-77.

Rosenbaum, J. L. (1989). Family dysfunction and female delinquency. Crime and Delinquency, 35 (1), 31-44.

Strauss, M. A. (1991). Discipline and deviance: Physical punishment of children and violence and other crime in adulthood. Social-Problems, 38 (2), 133-154.

Xiaoming, L., Feigelman, S. \& Stanton, B. (2000). Perceived parental monitoring and health risk behaviours among urban low-income african-american children and adolescents. Journal of Adolescents Health, 27, 43-48. 